\title{
Voir la pharmacie du point de vue de la santé publique
}

\author{
par Neil J MacKinnon
}

$\mathrm{D}$ epuis 1989, une partie importante de ma vie a été liée à la profession de pharmacien. À mesure que j’ai obtenu mes grades universitaires de premier cycle et de cycles supérieurs en pharmacie, travaillé comme pharmacien hospitalier et pharmacien communautaire, agi à titre de membre du corps professoral d'un collège de pharmacie, puis travaillé au sein d'organisations de pharmacie comme la SCPH, même mes amis non-pharmaciens savaient que la pharmacie était une grande partie de mon identité.

Tout cela a changé jusqu’à un certain point l'été dernier lorsque notre famille a déménagé à Tucson, en Arizona, et que je suis devenu professeur de santé publique à l'Université de l'Arizona et directeur de son centre de santé rurale (www.crh. arizona.edu/). Bien sûr, je suis autant pharmacien aujourd'hui que je l'étais il y a un an, mais pour la première fois en plus de 23 ans, je ne suis pas entouré de pharmaciens et d'éducateurs en pharmacie, et la plupart de mes activités ne sont pas liées à des questions de pharmacie. Je donne un cours de cycles supérieurs sur les politiques de santé rurale ce semestre-ci. Notre centre abrite le State Office of Rural Health, et par l'intermédiaire de ce bureau je travaille sur diverses questions de santé en milieu rural, notamment les services médicaux d'urgence, la sécurité des patients et l'amélioration de la qualité dans les hôpitaux en région rurale, la santé des Amérindiens et l'évaluation de la santé communautaire. L'expérience a été fascinante jusqu’à aujourd'hui et, en tant que pharmacien, j'estime que je peux offrir un point de vue unique sur ces questions et y contribuer véritablement.

D'une certaine façon, voir notre profession de l'extérieur a été une expérience extracorporelle. Du coup, je suis convaincu plus que jamais de la valeur que le pharmacien apporte à chaque patient individuellement et à la société dans son ensemble.

Du point de vue de la santé publique, le pharmacien peut participer de diverses façons, allant des programmes de vaccina- tion à la prise en charge et à la prévention des maladies, en passant par l'éducation pour la santé. La SCPH reconnait ce domaine comme l'un des six buts du projet SCPH 2015 : "Accroître le degré d'intervention des départements de pharmacie des établissements de santé dans la mise en œuvre d'initiatives de santé publique " (http://www.cshp.ca/programs/ cshp2015/index_e.asp).

Ici, aux États-Unis, le bureau du pharmacien en chef a soumis dernièrement un rapport pertinent au chef du service fédéral de la santé publiquel. Ce rapport discute du rôle utile que jouent les pharmaciens, et note que " les pharmaciens sont remarquablement sous-utilisés par le système de soins de santé étasunien, si l'on tient compte de leur niveau d'éducation, de leur formation et de leur proximité avec la collectivité. » De plus, le rapport stipule que l'un de ces quatre principaux objectifs est de " progresser au-delà des discussions (et des nombreux projets pilotes) sur l'élargissement du rôle des pharmaciens dans la prestation des soins aux patients et passer à leur mise en oeuvre dans le système de santé. »

Alors, mettons tous la main à la pâte afin de veiller à ce que nos compétences soient correctement utilisées et mises en œuvre... au profit de chaque patient et de la santé publique.

[Traduction par l'éditeur]

\section{Référence}

1. Giberson S, Yoder S, Lee MP. Improving patient and health system outcomes through advanced pharmacy practice. A report to the U.S. Surgeon General 2011. US Public Health Service, Office of the Chief Pharmacist; décembre 2011. Publié au www.usphs.gov/corpslinks/pharmacy/documents/ 2011AdvancedPharmacyPracticeReporttotheUSSG.pdf. Consulté le 23 mai 2012.

Neil J MacKinnon, B. Sc. (Pharm.), M. Sc. (Pharm.), Ph. D., FCSHP, est président sortant et agent de liaison pour la vision de la SCPH. 